\title{
MDM transmission using air-clad photonic lanterns
}

Mariam Mathew, Neethu; Grüner-Nielsen, Lars; Galili, Michael; Lillieholm, Mads; Rottwitt, Karsten

Published in:

IEEE Photonics Technology Letters

Link to article, DOI:

10.1109/LPT.2020.3011183

Publication date:

2020

Document Version

Peer reviewed version

Link back to DTU Orbit

Citation (APA):

Mariam Mathew, N., Grüner-Nielsen, L., Galili, M., Lillieholm, M., \& Rottwitt, K. (2020). MDM transmission using air-clad photonic lanterns. IEEE Photonics Technology Letters, 32(17), 1049 - 1052.

https://doi.org/10.1109/LPT.2020.3011183

\section{General rights}

Copyright and moral rights for the publications made accessible in the public portal are retained by the authors and/or other copyright owners and it is a condition of accessing publications that users recognise and abide by the legal requirements associated with these rights.

- Users may download and print one copy of any publication from the public portal for the purpose of private study or research.

- You may not further distribute the material or use it for any profit-making activity or commercial gain

- You may freely distribute the URL identifying the publication in the public portal

If you believe that this document breaches copyright please contact us providing details, and we will remove access to the work immediately and investigate your claim 


\title{
MDM transmission using air-clad photonic lanterns
}

\author{
Neethu Mariam Mathew, Lars Grüner-Nielsen, Member, IEEE, Michael Galili, Mads Lillieholm, and Karsten \\ Rottwitt
}

\begin{abstract}
MIMO less few mode transmission is performed using two spatial channels transmitted through $1 \mathrm{~km}$ of two mode graded index fiber at $10 \mathrm{~Gb} / \mathrm{s}$ modulation rate. The multiplexing and de-multiplexing of the spatial channels are done using air clad photonic lanterns. The power in the two degenerate $L P_{11}$ modes are added electrically and counted as a single channel. The $Q$ values calculated for the $L P_{01}$ and $L P_{11}$ channels are 5.4 and 4.7 respectively. We also show that, the signal degradation due to modal interference can be mitigated by using different laser sources for different spatial channels with some wavelength spacing. The wavelength separation increases the modal beat frequency beyond the detector bandwidth, thus improving the transmission performance. Using two laser sources with a wavelength spacing of $0.2 \mathrm{~nm}$ between the spatial modes could improve the $Q$ compared to the case where a single laser source is split between the spatial channels with some extra length of fiber in one of the paths for optical decorrelation.
\end{abstract}

Index Terms-Optical fiber devices, Optical fiber communication, Intensity modulation

\section{INTRODUCTION}

$\mathbf{M}$ ODE division multiplexing (MDM) using few mode fibers has been a very active research field in the last decade. Usually, coherent detection and multiple input multiple output (MIMO) digital signal processing is used to mitigate the modal crosstalk in these few mode transmission systems. However, MIMO processing is computationally complex and energy consuming which limits the practical applications of MDM. There are ways to reduce this complexity. For example, in a weakly coupled Few Mode Fiber (FMF), the inter channel distortion due to mode coupling is reduced by increasing the effective index difference between the linearly polarized (LP) modes in the fiber. This could reduce the MIMO complexity to $2 \times 2$ MIMO for the non degenerate modes and $4 \times 4$ MIMO for the degenerate modes [1]. If the two polarization channels and the degenerate spatial channels are not separated, and counted as a single channel, then MIMO processing can be fully avoided. The interests in this kind of MDM without MIMO has emerged lately in short reach applications like data center networks and interconnections for high performance computers [2] [3]. Conventional intensity modulation and direct detection (IM-DD) are preferred in these short reach applications due to the simple structure and low cost. MDM without MIMO requires mode multiplexers that can specifically excite the required spatial mode in the fiber, and mode de-multiplexers that can transform light back from different

N. M. Mathew, e-mail: namama@fotonik.dtu.dk, L. Grüner-Nielsen, M. Galili, M. Lilleholm and K. Rottwitt are with DTU Fotonik, Technical University of Denmark, Ørsteds Plads, Building 343, 2800 Kgs. Lyngby, Denmark

L. Grüner-Nielsen is also with Danish Optical Fiber Innovation, Åvendingen 22A, 2700 Brønshøj, Denmark.

Manuscript received April 19, 2005; revised August 26, 2015. higher order modes. Fiber based mode selective couplers (MSC) can perform these functions and IM-DD transmission using such couplers has been reported [4]. Mode selective couplers excite one LP mode at a time. Thus for each LP mode group a separate MSC is required. Furthermore, MSC can only detect one of the degenerate modes in a specific mode group. A mode selective photonic lantern can overcome this limitation as it can excite different LP modes in a FMF at the same time. The power in both degenerate modes in the FMF can be also be simultaneously detected. Mode selective photonic lanterns that can excite up to 15 spatial modes has been reported [5]. $3 \times 10 \mathrm{~Gb} / \mathrm{s}$ mode group transmission over $20 \mathrm{~km}$ of FMF has been demonstrated using five port photonic lanterns. [6]. We have earlier reported the fabrication and characterization of an air cladded mode group selective lantern that could excite the first two LP mode groups [7]. These air cladded lanterns are fabricated without the use of the fluorine doped low index outer cladding which surrounds the single mode fibers (SMFs) in the usual fabrication technique. In our technique, one standard SMF (SSMF) and two Corning HI1060 (smaller core diameter of $5.3 \mu \mathrm{m}$ ) fibers are stranded together and tapered down. The tapered fiber bundle is cleaved and spliced to an OFS Two Mode Step Index (TMSI) fiber. TMSI fiber is preferred here as it has larger mode field diameters for the $\mathrm{LP}_{01}$ and $\mathrm{LP}_{11}$ modes compared to the graded index fiber used for transmission and hence the single mode fiber bundle does not have to be tapered down below $22 \mu \mathrm{m}$.

In this paper we report the results of the MDM transmission using two fabricated lanterns. MIMO less $2 \times 10 \mathrm{~Gb} / \mathrm{s}$ transmission over $1 \mathrm{~km}$ of FMF and $2 \times 25 \mathrm{~Gb} / \mathrm{s}$ transmission over $7 \mathrm{~m}$ of FMF are reported here. We also show that the signal degradation due to modal interference can be reduced by using different lasers with a small wavelength spacing for the two spatial channels [8].

\section{MDM TRANSMISSION}

The experimental set up used for data transmission is shown in figure 1. A DFB laser is modulated using a Mach Zehnder modulator to generate a non-return-to-zero signal. A pseudo random bit sequence (PRBS) of length of $2^{31}-1$ is used for transmission. The amplified signal is split into two paths using a $3 \mathrm{~dB}$ coupler. The laser has a line width of a few $\mathrm{MHz}$, corresponding to a coherence length of around $100 \mathrm{~m}$. A $2 \mathrm{~km}$ $\mathrm{SMF}+300 \mathrm{~m}$ dispersion compensation fiber is included in one of the paths to decorrelate the optical paths. The two paths are separately amplified and fed into the $\mathrm{LP}_{01}$ and $\mathrm{LP}_{11}$ inputs of the multiplexing lantern. Variable attenuators are connected to each paths to balance the powers in the two modes, so the received power is the same in both channels. There is 7 


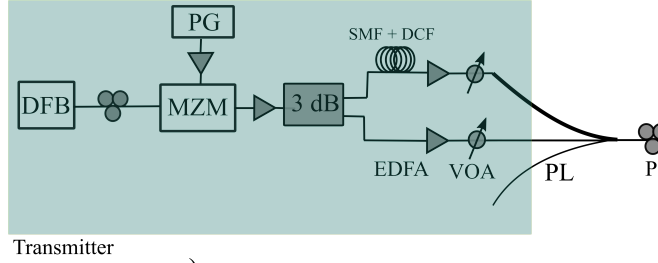

a)
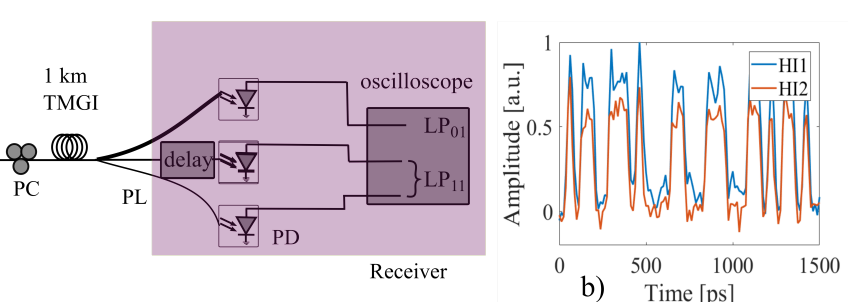

Fig. 1. a) Setup used for MDM transmission using two LP channels at $10 \mathrm{~Gb} / \mathrm{s}$ and $25 \mathrm{~Gb} / \mathrm{s}$. DFB: DFB laser, PG: Pattern Generator, MZM: Mach Zehnder Modulator, 3 dB: 3 dB splitter, EDFA: Erbium Doped Fiber Amplifier, VOA: Variable Optical Attenuator, PL: Photonic Lantern, PC: Polarization controller, TMGI: Two Mode Graded Index fiber, PD: Photo Diode. b) The alligned LP $_{11}$ traces before adding.

$\mathrm{m}$ of TMSI fiber between the two lanterns, and a polarization controller is set on the TMSI fiber. A $1 \mathrm{~km}$ long OFS two mode graded index fiber (TMGI) is also spliced between the two lanterns. Depending on the input polarization, the orientation of the $\mathrm{LP}_{11}$ mode in the FMF changes and hence the overlap with the lantern modes of the de-multiplexing lantern also changes. The polarization controller at the TMSI fiber can rotate the $\mathrm{LP}_{11}$ mode to get the lowest de-multiplexing cross talk. At the output lantern, the SSMF shown in thick line, delivers the de-multiplexed $\mathrm{LP}_{01}$ power. The two small core HI1060 fibers, each give an orthogonal linear combination of powers in $\mathrm{LP}_{11 a}$ and $\mathrm{LP}_{11 b}$ modes. Hence the total $\mathrm{LP}_{11}$ power is the sum of the two HI1060 outputs. Three photodiodes detect the output powers and the signals are acquired by an oscilloscope. The powers from the two HI1060 fibers are electrically added in the oscilloscope to get the total power in the $\mathrm{LP}_{11}$ channel. A free space delay is introduced at one HI1060 fiber to align the traces together before they are added, alternatively this delay could be added in the electrical signal processing by finding the delay that gives the maximum correlation between the traces, and shifting one of the traces accordingly. An example of the aligned output traces from the two HI1060 fibers for a modulation rate of $25 \mathrm{~Gb} / \mathrm{s}$ is shown in Figure 1b. The sampling speed of the oscilloscope is 80 $\mathrm{GS} / \mathrm{s}$ and the bandwidth is $33 \mathrm{GHz}$. The real time traces from the three output fibers are saved and there are $10^{6}$ points in each trace.

The polarization dependent crosstalk for the multiplexing lantern is measured using $\mathrm{S}^{2}$ set up as $12 \mathrm{~dB}-16.1 \mathrm{~dB}$ for the $\mathrm{LP}_{01}$ mode and $12.4 \mathrm{~dB}-14.9 \mathrm{~dB}$ for the $\mathrm{LP}_{11}$ mode [9]. For the demultiplexing lantern, when $\mathrm{LP}_{01}$ mode is launched to the TMSI fiber, the PDL for $\mathrm{LP}_{01}$ and $\mathrm{LP}_{11}$ modes are 2.6 $\mathrm{dB}-2.7 \mathrm{~dB}$ and $14.2 \mathrm{~dB}-15.2 \mathrm{~dB}$ respectively and when $\mathrm{LP}_{11}$ mode is launched into the TMSI fiber, the PDL for the $\mathrm{LP}_{01}$ and $\mathrm{LP}_{11}$ modes are $12 \mathrm{~dB}-20 \mathrm{~dB}$ and $3.3 \mathrm{~dB}-4.4$ $\mathrm{dB}$ respectively. The polarization dependent mode crosstalk matrix for the two lanterns connected back to back with $7 \mathrm{~m}$ of TMSI fiber between them is shown in table I. The splice between the TMSI fibers introduce some extra mode coupling here.

The transmission quality is assessed from the $\mathrm{Q}$ value of the output traces evaluated from the intensity distributions and standard deviations of the 1 and 0 bits. The data is transmitted simultaneously through the $\mathrm{LP}_{01}$ and $\mathrm{LP}_{11}$ channels at $10 \mathrm{~Gb} / \mathrm{s}$ and $25 \mathrm{~Gb} / \mathrm{s}$. The frequencies present in the output traces are first determined using a Fourier transform. The Fourier
TABLE I

THE MODE CROSSTALK MATRIX WHEN TWO LANTERNS ARE CONNECTED BACK TO BACK WITH 7 M OF TMSI BETWEEN THEM

\begin{tabular}{|c|c|c|}
\hline & output $\mathrm{LP}_{01}[\mathrm{~dB}]$ & output $\mathrm{LP}_{11}[\mathrm{~dB}]$ \\
\hline Input $\mathrm{LP}_{01}$ & $2.5-4.2$ & $6.9-12.7$ \\
\hline Input $\mathrm{LP}_{11}$ & $11.4-19.5$ & $4.8-6.4$ \\
\hline
\end{tabular}

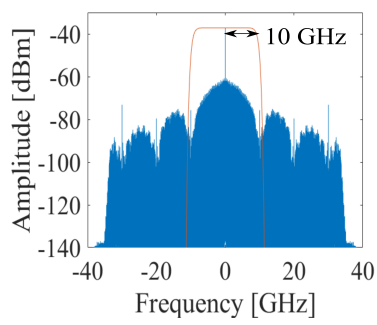

Fig. 2. The frequencies present in the saved $\mathrm{LP}_{01}$ trace with a modulation rate of $10 \mathrm{~Gb} / \mathrm{s}$. The Gaussian filter used on the output trace is shown in orange line.

transform of the $\mathrm{LP}_{01}$ channel trace, for $10 \mathrm{~Gb} / \mathrm{s}$ modulation rate is shown in figure 2. As the receiver bandwidth is much larger than the modulation frequency, a Gaussian filter is used on the saved traces. A nearly ideal 16th order super Gaussian filter is used to emulate the receiver bandwidth. The filter is shown in orange line in figure 2. The $\mathrm{Q}$ values are calculated at different filter bandwidths and shown in figure 3. These are for the $\mathrm{LP}_{01}$ channel at modulation rates of 10 $\mathrm{Gb} / \mathrm{s}$ and $25 \mathrm{~Gb} / \mathrm{s}$. It can be seen that the best transmission performance is obtained when the receiver bandwidth is close to the modulation frequency. Hence the bandwidth of the Gaussian filter is set same as the modulation frequency and the eye diagrams and $\mathrm{Q}$ values are evaluated for each channel. The evaluated $\mathrm{Q}$ values and the corresponding eye diagrams for the $\mathrm{LP}_{01}$ and $\mathrm{LP}_{11}$ channels are shown in figure $4 \mathrm{a}$ for a modulation rate of $10 \mathrm{~Gb} / \mathrm{s}$ and in figure $4 \mathrm{~b}$ for $25 \mathrm{~Gb} / \mathrm{s}$. Here, there is $7 \mathrm{~m}$ of TMSI between the multiplexing and demultiplexing lanterns. The $\mathrm{Q}$ values of the input signal fed into the multiplexing lantern is also shown for comparison. For 10 $\mathrm{Gb} / \mathrm{s}$, the $\mathrm{Q}$ values are high, but for $25 \mathrm{~Gb} / \mathrm{s}$ the $\mathrm{Q}$ value has reduced showing signal degradation.

As the next step, a $1 \mathrm{~km}$ TMGI fiber is spliced between the two lanterns. The TMGI fiber is preferred over the TMSI fiber because it has a much lower distributed mode coupling of -25 $\mathrm{dB}$ for a $30 \mathrm{~km}$ length [10]. Data is transmitted simultaneously 


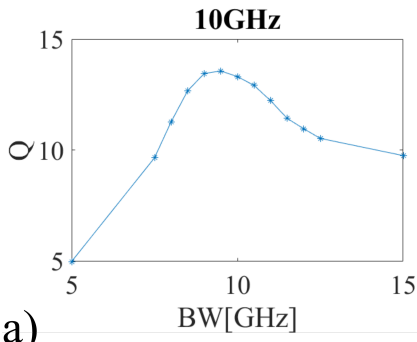

a)

$\mathrm{BW}[\mathrm{GHz}]$

Fig. 3. $\mathrm{Q}$ values for the $\mathrm{LP}_{01}$ channel at different receiver bandwidth at modulation rates of a) $10 \mathrm{~Gb} / \mathrm{s}$ and b) $25 \mathrm{~Gb} / \mathrm{s}$.

a)

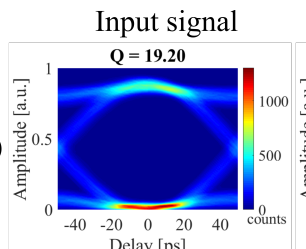

$Q=11.19$

b)

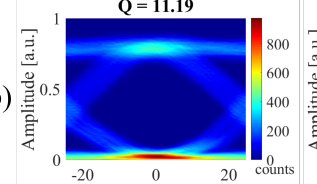

Delay [ps] $\mathbf{Q}=\mathbf{1 3 . 3 7}$

c)
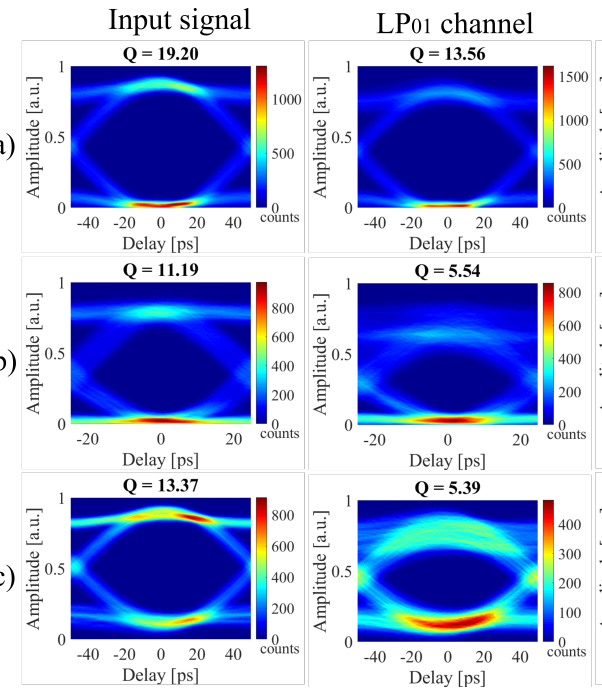

$\begin{array}{ccc}-20 & 0 & 20 \\ \text { Delay } & {[\mathrm{ps}]}\end{array}$
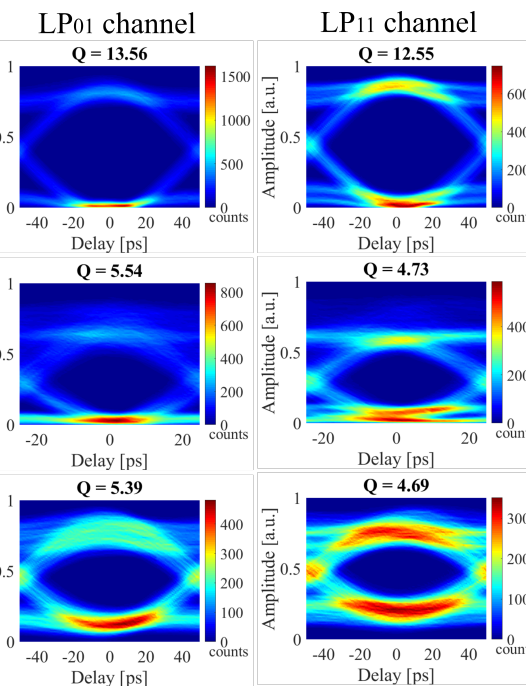

$Q=4.73$

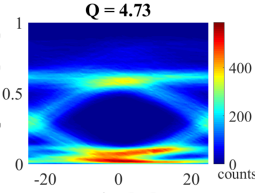

Delay [ps]

$\mathrm{Q}=\mathbf{4 . 6 9}$

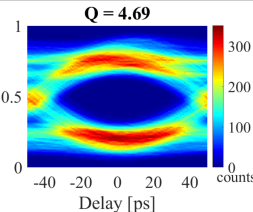

Fig. 4. Evaluated eye diagrams for the input signal and the two spatial channels for $7 \mathrm{~m}$ long transmission at a modulation frequency of a) $10 \mathrm{~Gb} / \mathrm{s}$ b) $25 \mathrm{~Gb} / \mathrm{s} \mathrm{c}$ ) for $1 \mathrm{~km}$ long transmission at $10 \mathrm{~Gb} / \mathrm{s}$.

through the two channels at $10 \mathrm{~Gb} / \mathrm{s}$. The calculated Q and the eye diagrams for this $1 \mathrm{~km}$ transmission is shown in figure $4 \mathrm{c}$. It is seen that for $1 \mathrm{~km}$ transmission, the $\mathrm{Q}$ value obtained is smaller than for the $7 \mathrm{~m}$ transmission shown in figure $4 \mathrm{a}$ at the same modulation rate of $10 \mathrm{~Gb} / \mathrm{s}$. The $\mathrm{LP}_{11}$ mode rotates along the length of the fiber and its overlap with the lantern modes in the demultiplexing lantern changes. This changes the polarization dependent de-multiplexing crosstalk as well. The longer the fiber, the faster this change will be. Hence, the polarization controller used on the FMF shown in figure 1 cannot be tuned fast enough for $1 \mathrm{~km}$ transmission. Mode coupling in the two splices between the TMSI and TMGI fibers also affects the transmission performance here.

Further investigation shows that there are two sources for signal degradation in this MIMO less MDM transmission. One is the pure crosstalk where the field in one spatial mode adds itself to the field in the other mode, and the other is the interference where the modes beat with each other. This modal noise is worse when there are only two modes in the fiber [11]. Usually, in the laboratory set ups this modal noise is reduced by using a delay in one of the spatial paths by connecting some extra fiber whose length exceeds the coherence length of the source. Another way to mitigate this modal interference is by using different wavelengths for the two modes, where the wavelength spacing is large enough that the beat frequency becomes much larger than the detector bandwidth. This kind of wavelength interleaving to mitigate modal crosstalk in FMF transmssions has already been reported [2] [12] [13]. In the next section we include an experimental study of these modal interference effects on FMF transmission.

\section{A. MDM transmission using two lasers}

To study the interference effects on FMF transmission, two laser sources are included in the experimental setup as shown in figure 5. Here, the two DFB lasers are separately modulated and used for the two spatial channels. One laser is set at a fixed wavelength of $1548.4 \mathrm{~nm}$, and the other laser is tuned so that the wavelength spacing is $0.2 \mathrm{~nm}$ Here we note that there is no wavelength filter on the photodiode as in the WDM transmission. The modulation frequency is $10 \mathrm{~Gb} / \mathrm{s}$ and the modulation format is non-return-to-zero. There is $7 \mathrm{~m}$ of FMF between the two lanterns. The evaluated eye diagrams and $Q$ values for the two channels are shown in figure 6a. The polarization dependent crosstalk for the demultiplexing lantern is $4 \mathrm{~dB}$ to $5 \mathrm{~dB}$ higher than that of the lantern used in the set up in section II. In addition, in this set up, the MZM modulator used to modulate the DFB laser for the $\mathrm{LP}_{11}$ channel is not as good as that of the modulator used for $\mathrm{LP}_{01}$ channel. This could degrade the $\mathrm{Q}$ values of the output traces. Hence, the $\mathrm{Q}$ values evaluated for the input signals to both channels are given in figure 6 for reference. This FMF transmission is also repeated using a single laser, where a $1.7 \mathrm{~km}$ SMF is connected in one of the input paths. The corresponding $\mathrm{Q}$ values are shown in figure $6 \mathrm{~b}$. Comparing the results, it is seen that, using wavelength interleaving between the spatial modes can improve the $\mathrm{Q}$ value relative to using a single laser source and optical decorrelation in one of the paths. The wavelength spacing of $0.2 \mathrm{~nm}$ makes the modal beating much faster than the receiver bandwidth. This improves the transmission performance as seen in the evaluated $Q$ values for the two cases.

\section{DISCUSSION}

In the above, we show that the fabricated air clad photonic lanterns could be used for MIMO less MDM transmission for short range applications. In [6], the powers from the two degenerate modes are combined optically using a third lantern, while in this work, the powers in the degenerate modes are combined in the electrical domain. In section II, a single laser source is used for transmission. $10 \mathrm{~Gb} / \mathrm{s}$ and $25 \mathrm{~Gb} / \mathrm{s}$ modulation rates are tried on $7 \mathrm{~m}$ long TMSI fiber. For $25 \mathrm{~Gb} / \mathrm{s}$ there is signal degradation due to the crosstalk in the lantern. $1 \mathrm{~km}$ long TMGI transmission is also tried at $10 \mathrm{~Gb} / \mathrm{s}$. The polarization dependent crosstalk in the fabricated lanterns is one of the main limiting factor for $1 \mathrm{~km}$ transmission. A follow up of this work would be to optimize the fabrication process to develop better lanterns with smaller crosstalk and polarization dependence and try longer MDM transmission with them. In section II-A, we show that signal degradation due to modal interference can be mitigated by using different laser sources with some wavelength spacing. The lanterns used in 


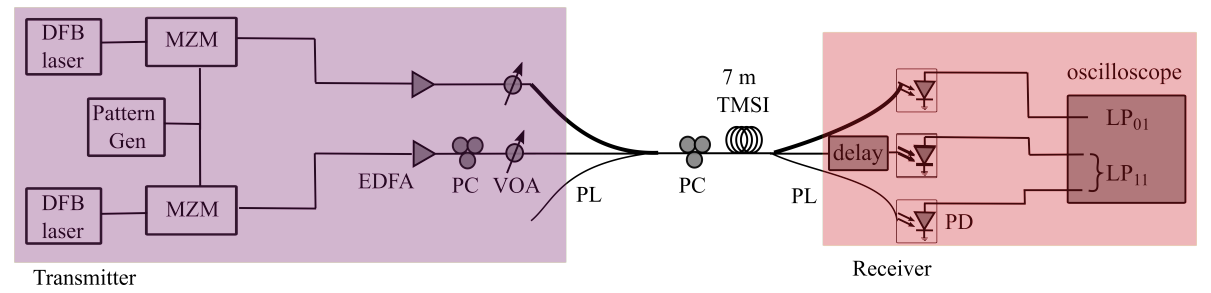

Fig. 5. Transmission setup with two lasers. MZM: Mach Zender Modulator, EDFA: Erbium Doped Fiber Amplifier, PC: Polarization Controller, VOA: Variable Optical Attenuator, PL: Photonic Lantern, TMSI:Two Mode Step Index fiber, PD: Photodiode.

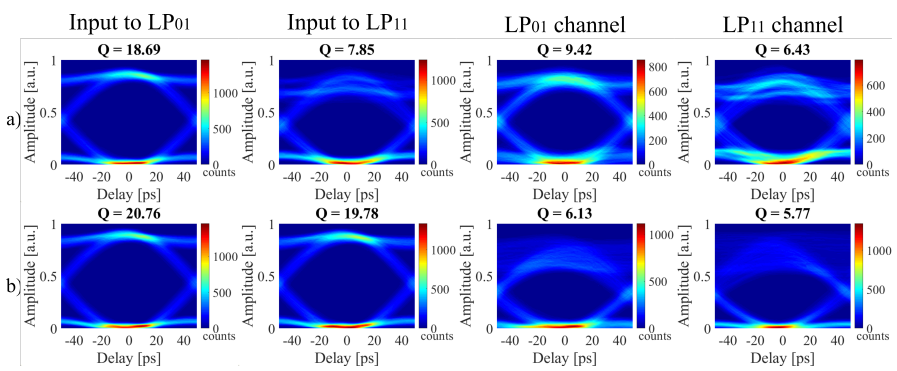

Fig. 6. Evaluated eye diagrams for the input signals and two spatial channels for $7 \mathrm{~m}$ long transmission at $10 \mathrm{~Gb} / \mathrm{s}$ data rate using a) two lasers with 0.2 $\mathrm{nm}$ wavelength spacing between them and $\mathrm{b}$ ) a single laser.

this part of the experiment have higher crosstalk, hence the transmission is done only at $10 \mathrm{~Gb} / \mathrm{s}$. For $10 \mathrm{GHz}$ receiver band width, a wavelength spacing of $0.2 \mathrm{~nm}$ could significantly improve the transmission performance compared to the case where a single laser source is used, where the modal inference degrades the performance of both channels. This kind of wavelength interleaving is better than using a delay on one of the paths using a decorrelation fiber to mitigate the modal interference in transmission systems. Numerical studies shows that for a detector bandwidth of $10 \mathrm{~Gb} / \mathrm{s}$ a minimum wavelength spacing of $0.2 \mathrm{~nm}$ is needed to mitigate the interference noise due to modal beating [14]. A more systematic experimental study of different wavelength spacing is difficult, as both the lantern crosstalk and the the modal interference are polarization dependent.

\section{CONClusion}

The multiplexing and demultiplexing capabilities of mode selective air clad photonic lanterns are tested using $2 \times$ $10 \mathrm{~Gb} / \mathrm{s}$ and $2 \times 25 \mathrm{~Gb} / \mathrm{s}$ MIMO-less MDM transmission. The evaluated $Q$ values for $1 \mathrm{~km}$ transmission at $10 \mathrm{~Gb} / \mathrm{s}$ through a two mode graded index fiber for the $\mathrm{LP}_{01}$ and $\mathrm{LP}_{11}$ channels are 5.4 and 4.7 respectively. We also show that the signal distortion due to modal interference can be mitigated by using different wavelengths for the two modes. For a receiver bandwidth of $10 \mathrm{GHz}$, a spacing of $0.2 \mathrm{~nm}$ could increase the $\mathrm{Q}$ compared to the case where a single laser is used.

Acknowledgment: The Danish council for independent research, grant DFF-4184-00433 "Signal processing using higher order modes in optical fibers", and Innovation fund Denmark, project 8057-00059B INCOM, DNRF Research
CoE, SPOC (DNRF123) are acknowledged for financial support.

\section{REFERENCES}

[1] P. Sillard, "Advances in few-mode fiber design and manufacturing," in Optical Fiber Communication Conference (OFC) 2020, p. W1B.4, Optical Society of America, 2020.

[2] L. Shen, D. Ge, Y. Liu, L. Xiong, S. Chen, H. Zhou, R. Zhang, L. Zhang, J. Luo, and J. Li, "Mimo-free $20-\mathrm{Gb} / \mathrm{s} \times 4 \times 2$ wdm-mdm transmission over 151.5-km single-span ultra low-crosstalk fmfs," in 2018 European Conference on Optical Communication (ECOC), p. Tu1G6, Sep. 2018.

[3] W. Wang, J. Zhao, L. Zhang, Q. Mo, Z. Yang, C. Li, Z. Wang, Z. Zhang, C. Carboni, and G. Li, "4 $\times 10$-gb/s mimo-free polarization and mode group multiplexing for data center applications," IEEE Photonics Technology Letters, vol. 29, no. 20, pp. 1711-1714, 2017.

[4] S. Jiang, C. Liang, L. Ma, J. Xiong, W. Zhang, and Z. He, "Ultra-lowloss broadband all-fiber mode selective couplers for mimo-less mdm transmission," J. Lightwave Technol., vol. 38, pp. 2376-2382, Apr 2020.

[5] A. Velázquez-Benítez, J. Antonio-López, J. alvarado zacarias, N. Fontaine, R. Ryf, H. Chen, J. Hernández-Cordero, P. Sillard, C. Okonkwo, S. Leon-Saval, and R. Amezcua-Correa, "Scaling photonic lanterns for space-division multiplexing," Scientific Reports, vol. 8, 2018.

[6] H. Liu, H. Wen, J. C. A. Zacarias, J. E. Antonio-Lopez, N. Wang, P. Sillard, R. Amezcua-Correa, and G. Li, "Demonstration of stable $3 \times 10 \mathrm{gb} / \mathrm{s}$ mode group-multiplexed transmission over a $20 \mathrm{~km}$ few-mode fiber," in Optical Fiber Communication Conference, p. W4J.2, Optical Society of America, 2018.

[7] N. M. Mathew, J. B. Christensen, L. Grüner-Nielsen, M. Galili, and K. Rottwitt, "Air-cladded mode-group selective photonic lanterns for mode-division multiplexing," Opt. Express, vol. 27, pp. 13329-13343, Apr 2019.

[8] N. M. Mathew, , L. Grüner-Nielsen, M. Galili, M. Lilleholm, and K. Rottwitt, "Cross talk and interference in mimo less few mode transmission systems," in 2020 CLEO (to be published), p. JW2E.24, May 2020.

[9] L. Grüner-Nielsen, N. M. Mathew, and K. Rottwitt, "Invited paper: Characterization of few mode fibers and devices," Optical Fiber Technology, vol. 52, p. 101972, 2019.

[10] L. Grüner-Nielsen, J. W. Nicholson, K. Jespersen, Y. Sun, R. Lingle, D. Jacobsen, and B. Pálsdóttir, "Measuring distributed mode scattering in few mode fibers with high and low differential group delay," in 2012 IEEE Photonics Society Summer Topical Meeting Series, pp. 193-194, 2012.

[11] R. E. Epworth, "Phenomenon of modal noise in fiber systems," in Optical Fiber Communication, p. ThD1, Optical Society of America, 1979.

[12] Y. Tian, J. Li, Z. Wu, P. Zhu, Y. Chen, Q. Mo, F. Ren, Z. Li, Y. He and Z. Chen, "Im-dd mdm-wdm transmission over $120-\mathrm{km}$ weaklycoupled fmf enabled by wavelength interleaving," in Optical Fiber Communication Conference, p. Tu3I.1, Optical Society of America, 2017.

[13] K. Benyahya, C. Simonneau, A. Ghazisaeidi, N. Barré, P. Jian, J.-F. Morizur, G. Labroille, P. Sillard, J. Renaudier, and G. Charlet, " $5 \mathrm{tb} / \mathrm{s}$ transmission over $2.2 \mathrm{~km}$ of multimode om2 fiber with direct detection thanks to wavelength and mode group multiplexing," in Optical Fiber Communication Conference, p. M2D.2, Optical Society of America, 2017.

[14] L. Grüner-Nielsen, N. M. Mathew, and K. Rottwitt, "Modeling of mimo less mode division multiplexed systems," Submitted to Photonic technology letters, 2020. 